\title{
A structural quality evaluation model for three-dimensional simulations
}

https://doi.org/10.1515/phys-2019-0035

Received January 25, 2019; accepted February 18, 2019

\begin{abstract}
:
Purpose - In recent years, computer simulations have become an innovative approach which enables research in the field of highly complicated physical phenomena and the study of the laws which govern the universe. The proper interpretation of the results of a visual simulation requires the highest quality of the generated image, as every distortion or mistake may have a significant influence on the readability, accuracy and even credibility of the presentation of the results. The aim of this presentation is to determine a model that enables precise quality evaluation of the three-dimensional visual simulations in the field of structural correctness.
\end{abstract}

Design/methodology/approach - The developed model is a solution that makes it possible to estimate the quality of stereoscopic image in the context of major threedimensional structural dysfunctions, namely, vertical parallax, rotation mismatch and scale mismatch. Implementing the wrought theoretical model with the use of costeffective mechanisms of image feature detection creates a robust method which enables the scalar grade of structural correctness of the studied three-dimensional simulation to be computed.

Findings - On the basis of the conducted research, in particular, taking into account three-dimensional simulations, it was stated that the formulated model with the developed method provides an efficient, structural quality estimation tool applicable for a wide variety of threedimensional images. The obtained results indicate that the wrought method has huge potential in the application of high-resolution simulations by enabling a screening test of the structural quality of the stereoscopic view in quasireal time.

Practical implications - The developed method may be used both in order to verify the quality of ready-to-use three-dimensional image and also at the stage of the calibration of input parameters of the simulation.

Originality/value - The paper takes into account the selection of the most significant distortions which occur in visual, three-dimensional simulations providing a cost- effective and versatile tool which allows for the detection and elimination of serious mistakes and dysfunctions as early as at the calibration stage.

Keywords: 3D simulations, stereoscopic image distortions, structural quality evaluation model, three-dimensional simulation improvement

PACS: 42.66.Si, 42.30.Tz, 42.30.Va, 42.66.Ne

\section{Introduction}

The intensive development of software observed over recent years has provided many innovative solutions used commonly in scientific laboratories. Modern computer software provides a convenient research environment allowing for the saving of financial-resources, whilst ensuring a wide variety of simulation functionalities [1-3]. Realizing studies with the use of computer calculation environments is particularly common in the field of exploring physical properties and phenomena [4-6]. The mentioned issues of science commonly use modern technologies to create visual simulations of researched phenomena as it would be extremely expensive or difficult to recreate them in the lab $[7,8]$. Preliminary parametrization and calibration of the programming software environment is a key aspect in the simulation realization process [9]. The issues mentioned above are especially significant regarding three-dimensional visual simulations in which the correctness of the generated image has a direct influence on the ability to properly and precisely interpret the obtained results. In the case of three-dimensional simulations, which are based on stereoscopic mechanisms, there is a major risk of structural distortions appearing on an image. Consequently, there is a crucial need to provide tools that

Michał Kowalczyk: Institute of Information Technology, Lodz University of Technology, Łódź, Poland, E-mail: michal.kowalczyk@edu.p.lodz.pl

Piotr Napieralski: Institute of Information Technology, Lodz University of Technology, Łódź, Poland, E-mail: piotr.napieralski@p.lodz.pl 
would enable the precise evaluation of the simulation image quality.

\section{Three-dimensional simulation structural distortions}

Structural distortions of the image occurring in threedimensional simulations are mainly conditioned by the inappropriate parametrization of the acquisition tools which are employed. Incorrect settings of the simulation environment may contribute to increasing structural problems, such as vertical parallax, rotation mismatch and scale mismatch $[10,11]$.

\subsection{Vertical parallax}

Vertical parallax is a distortion of a three-dimensional image that means a mutual displacement of stereoscopic views occurs in the simulation in the vertical plane (Fig. 1, 2). The described problem is conditioned by the incorrect settings of the convergence or by the inappropriate alignment of the throwing areas of the stereoscopic views of the image in the vertical plane.
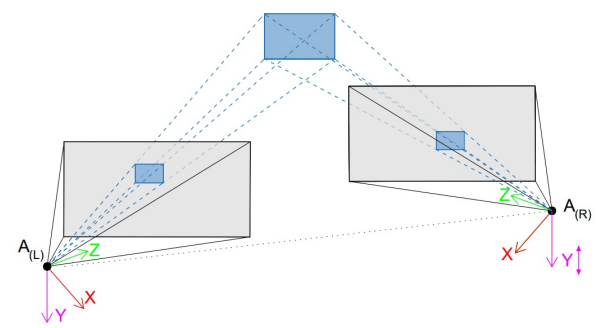

Figure 1: Scheme model of the throwing area of three-dimensional views of the simulation affected by vertical parallax distortion.

\subsection{Rotation mismatch}

Rotation mismatch is a distortion of a stereoscopic image that consists in a mutual rotation of the stereopair views occurring with respect to the axis of the depth of the threedimensional simulation (Fig. 3, 4). The rotation deformation stems from the incorrect settings of the stereoscopic view throwing area coherence with regard to the axis of the image depth.

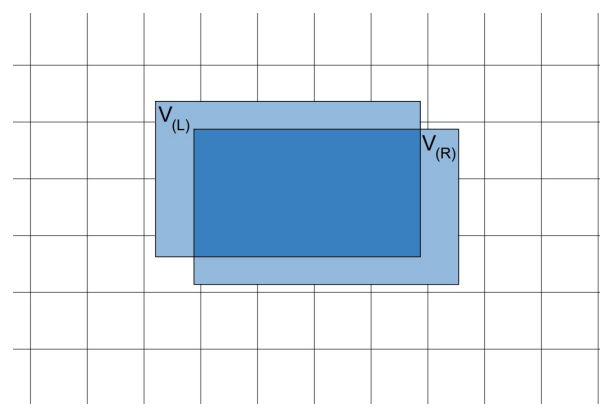

Figure 2: Model of the anaglyph image of a three-dimensional simulation affected by vertical parallax distortion.

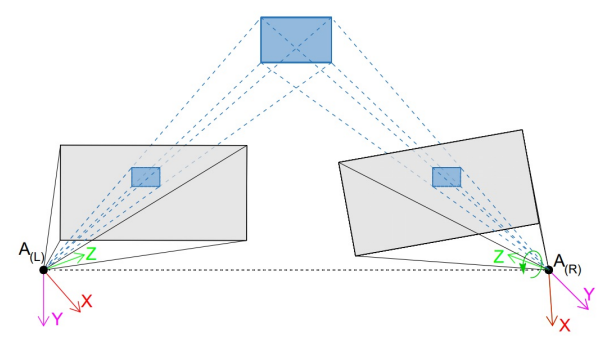

Figure 3: Scheme model of the throwing area of three-dimensional views of the simulation affected by rotation mismatch distortion.

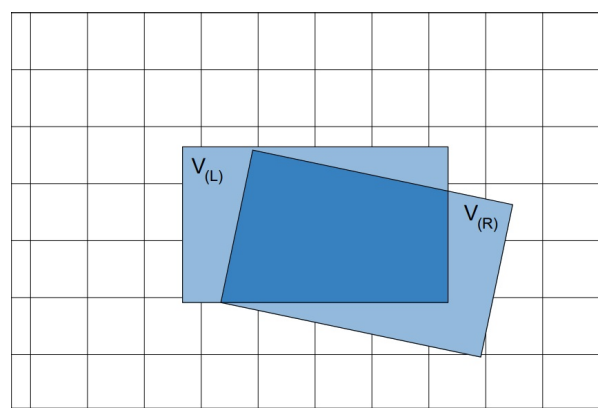

Figure 4: Model of the anaglyph image of a three-dimensional simulation affected by rotation mismatch distortion.

\subsection{Scale mismatch}

Scale mismatch of a three-dimensional simulation image is a distortion meaning the size inconsistency of rendered objects occurring in the left and right stereoscopic view (Fig. 5, 6). The scaling problem is conditioned by the inconsistent setting of the stereoscopic views' throwing area placement with respect to the depth coordinate. This, in turn, has an impact on making the stereopair perspective inconsistent which means incorrect object sizes presented on the steroviews. 


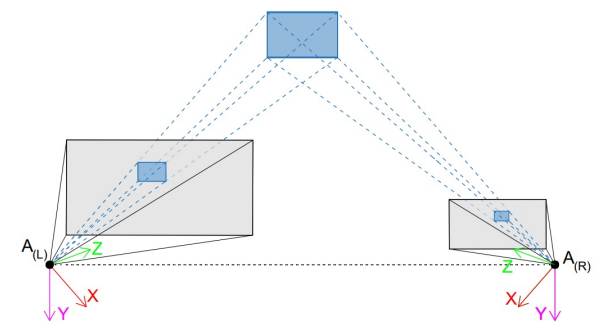

Figure 5: Scheme model of the throwing area of three-dimensional views of a simulation affected by scale mismatch distortion.

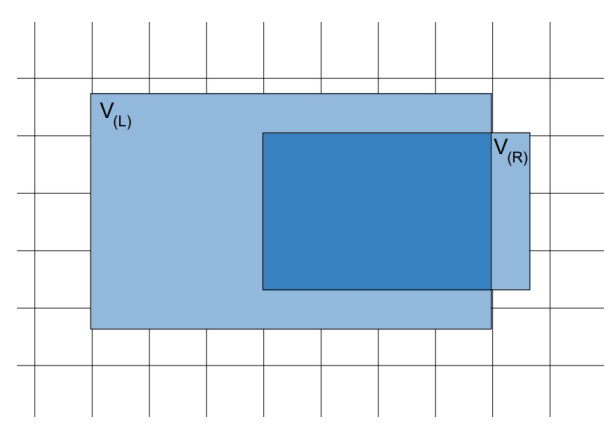

Figure 6: Model of the anaglyph image of a three-dimensional simulation affected by scale mismatch distortion.

\section{Structural distortion measurements}

Estimating the values of structural distortions in threedimensional simulations requires the calculation of geometric dependents between the stereopair views ( $l$ - left and $r$ - right) of the considered image. Most solutions applied for this purpose use direct correlation methods of views, which turns out to be too time-consuming in calculations for $4 \mathrm{~K}$ high-resolution images, such as threedimensional simulations. Within the framework of the realized research, it was decided to use the modified method based on the detection of image features which enables efficient point matching of high-resolution stereoscopic views whilst ensuring the high quality of the generated results thanks to the use of the sliding window mechanism [12]. The reference method has been modified by an additional mechanism of filtering, which consists in the selection of matchings with respect to the smallest limit vector distance. It provides an efficient solution which allows a low-density map of stereopair views correlations $N$ points $\left\{P_{\left(x_{l}, y_{l}\right)_{N}}\right\},\left\{P_{\left(x_{r}, y_{r}\right)_{N}}\right\}$ with consistent distribution and high-quality matchings to be obtained.

With information about the point correlation of the stereoscopic views, it is possible to calculate the numer- ical values of the structural distortions of images on the basis of the presented measurements (eq.1-3).

\subsection{Vertical parallax}

The value of an average vertical parallax $\Delta$ of the simulation image can be calculated as the arithmetic average of the subtraction of vertical coordinates of $N$ correlated key points $\left\{P_{\left(x_{l}, y_{l_{N}}\right\}}\right\},\left\{P_{\left(x_{r}, y_{r}\right)_{N}}\right\}$ of the stereopair (eq.1).

$$
\Delta\left(\left\{P_{\left(x_{l}, y_{l}\right)_{N}}\right\},\left\{P_{\left(x_{r}, y_{r}\right)_{N}}\right\}\right)=\frac{\sum_{i=1}^{N} y_{l_{i}}-y_{r_{i}}}{N}
$$

\subsection{Rotation mismatch}

The value of an average rotation mismatch $\alpha$ of the simulation image is calculated as an arithmetic mean of consecutive values of rotation mismatch defined in individual $N$ key points. The value of rotation mismatch at the point can be calculated as the arctg of the angles between chosen pair of corresponding straight lines.

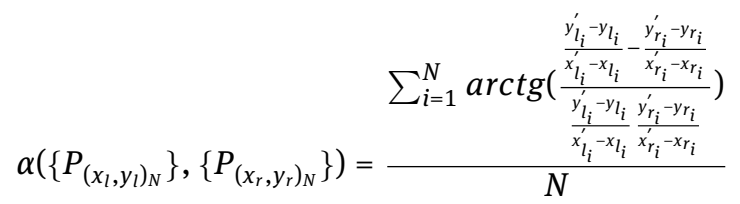

The straight lines are defined between the considered point and the one of a remaining point, both in the left and in the right stereoscopic view, creating corresponding pairs of straight lines. The process is repeated for all $N$ points. Calculated in that way, the $N$ values are averaged to obtain the final value of rotation mismatch of the whole image (eq.2).

\subsection{Scale mismatch}

The average scale mismatch value $s$ of the simulation image is calculated analogously to the value of the average rotation mismatch.

$$
s\left(\left\{P_{\left(x_{l}, y_{l}\right)_{N}}\right\},\left\{P_{\left(x_{r}, y_{r}\right)_{N}}\right\}\right)=\frac{\sum_{i=1}^{N} \frac{\sqrt{\left(x_{l_{i}}^{\prime}-x_{l_{i}}\right)^{2}+\left(y_{l_{i}}^{\prime}-y_{l_{i}}\right)^{2}}}{\sqrt{\left(x_{r_{i}}^{\prime}-x_{r_{i}}\right)^{2}+\left(y_{r_{i}}^{\prime}-y_{r_{i}}\right)^{2}}}}{N}
$$

The difference is that the arithmetic mean is computed on the basis of the quotient of the corresponding line segments lengths and defined between points in the left and 
right stereoscopic view. The final value of the scale mismatch is the arithmetic mean of the $N$ point values (eq.3).

\section{The structural quality evaluation model}

The aggregation of values of partial measurements of structural distortions allows for the development of a versatile model that evaluates the quality of threedimensional simulations.

The research literature provides many methods used for that purpose, but the normalized Minkowski norm (where $\mathrm{n}$ is the number of aggregated partial parameters) is favored as the leading solution (eq.4) [13].

$$
M=\sqrt[n]{\frac{\sum_{i=1}^{n}|P|^{n}}{n}}
$$

Formulating model $Q_{s}$, which enables an evaluation of the structural quality in three dimensional simulations, requires the normalization of three considered values $(n=3)$ : vertical parallax $\Delta$ in terms of the height of the applied sliding window $w$, rotation mismatch $\alpha$ with regard to the properties of function $\operatorname{arctg} m=90$, and scale mismatch $s$ in the range of $\langle 0,1\rangle$.

$$
Q_{s}=1-\sqrt[n]{\frac{\left|\frac{\Delta}{w}\right|^{n}+\left|\frac{\alpha}{m}\right|^{n}+|1-s|^{n}}{n}}
$$

The proposed model (eq.5) allows for an evaluation of the structural correctness of three-dimensional simulations in the scope of vertical parallax, rotation mismatch and scale mismatch, returning a scalar quality value in the range of $\langle 0,1\rangle$. A value of 0 means a high level of distortion while a value of 1 indicates that the three-dimensional simulation is devoid of considered mistakes.

\section{Tests}

The process of testing the structural quality evaluation model was realized by testing a set of three-dimensional simulations and reference values developed with the $3 \mathrm{ds}$ Max Studio 2016 application. The measurement of computational time was carried out with an Intel i5-7200U
$2.5 \mathrm{GHz}$ processor, ensuring a single separated processor thread.

The tests have been carried out for a couple of cases differing between each other in sliding window parameters. The below presented tables show the most significant results.

Table 1: Values of the structural distortions calculated in the testing process. A) name $L_{-}{ }^{*}$ - low resolution simulation $1920 \times 1080$ [px], $\mathrm{H}_{-}{ }^{*}$ - high resolution simulation $3840 \times 2160$ [px]; B) reference value of average vertical parallax[px]; C) developed value of average vertical parallax[px]; D) reference value of average rotation mismatch [deg]; E) developed value of average rotation mismatch [deg]; F) reference value of average scale mismatch; $G$ ) developed value of average scale mismatch;

\begin{tabular}{clllllc}
\hline A & \multicolumn{1}{c}{$B$} & \multicolumn{1}{c}{ C } & \multicolumn{1}{c}{ D } & E & F & G \\
\hline L_1 & 0.000 & 0.003 & -0.001 & 0.000 & 1.000 & 1.000 \\
H_1 & 0.001 & 0.005 & 0.001 & 0.002 & 1.000 & 1.000 \\
L_2 & 5.621 & 5.687 & 0.541 & 0.469 & 1.101 & 1.098 \\
H_2 & 11.245 & 11.321 & 0.538 & 0.464 & 1.102 & 1.100 \\
L_3 & 20.124 & 20.021 & 1.112 & 1.097 & 1.257 & 1.262 \\
H_3 & 40.481 & 40.381 & 1.113 & 1.100 & 1.254 & 1.256 \\
L_4 & 39.096 & 39.102 & 9.231 & 9.234 & 1.451 & 1.452 \\
H_4 & 78.012 & 78.304 & 9.233 & 9.234 & 1.449 & 1.450 \\
\hline
\end{tabular}

Table 2: Values of the structural quality calculated in the testing process. A) name $L_{-}{ }^{*}$ - low resolution simulation $1920 \times 1080$ [px], $\mathrm{H}_{-}{ }^{*}$ - high resolution simulation $3840 \times 2160$ [px]; H) reference value of structural quality; I) developed value of structural quality; J) calculation time[ms]

\begin{tabular}{cccc}
\hline A & H & I & J \\
\hline L_1 & 1.000 & 1.000 & 1981 \\
H_1 & 1.000 & 1.000 & 11681 \\
L_2 & 0.891 & 0.891 & 1892 \\
H_2 & 0.891 & 0.891 & 10471 \\
L_3 & 0.636 & 0.637 & 1898 \\
H_3 & 0.635 & 0.635 & 9945 \\
L_4 & 0.301 & 0.300 & 1886 \\
H_4 & 0.302 & 0.300 & 10241 \\
\hline
\end{tabular}




\section{Conclusions}

Simulations make it possible to study phenomena under restriction-free conditions without the risk that the results will be affected by factors that were unknown or impossible to eliminate in the research. However, only a simulation which is developed correctly and calibrated precisely can be a useful tool. This is especially important in three-dimensional simulations where various distortions can significantly affect the quality of the results.

Our proposed structural quality evaluation model aims to provide a simple method to evaluate the structural quality of three-dimensional simulations. Testing the proposed model shows that the results are extremely close to the reference values independent of the input image resolution (Tab. 1). The obtained results and calculation times indicate that the proposed model is an efficient and versatile method which makes it possible to precisely assess the structural quality of any stereoscopic material (Tab. 2). The developed tool has a wide variety of applications in different fields of science, as being able to evaluate the structural quality and, if necessary, refining every single aspect of the simulation to obtain a structural quality value close to 1 allows studies to achieve satisfying and reliable results.

\section{References}

[1] Guzek K., Napieralski P., Muszyński G., Wide Field of View Projection Using Rasterization, Multimedia and Network Information Systems, Proceedings of the 11th International Conference (MISSI 2018), Springer, 2019, pp. 586-595

[2] Bielak A., Bednarski R., Wojciechowski A., 3D Modeling of Leg Muscle Using Mechanochemical Representations of Muscular Tissue and Solid Fibers, Information Technology in Biomedicine, Springer, 2019
[3] Wawrzonowski M., Daszuta M., Szajerman D., Napieralski P., Mobile devices' GPUs in cloth dynamics simulation, Proceedings of 2017 Federated Conference on Computer Science and Information Systems (FedCSIS 2017), 2017, pp. 1283-1290

[4] Wiak S., Firych-Nowacka A., Smótka K., Computer models of 3D magnetic microfibres used in textile actuators, The international journal for computation and mathematics in electrical and electronic engineering, 2010, Vol. 29 Issue: 5, pp.1159-1171

[5] Zgraja J., Simplified simulation technique of rotating, induction heated, calender rolls for study of temperature field control, Open Physics, 2018, Vol 15, No. 1 pp. 326-331

[6] Smótka, K., Firych-Nowacka, A., Lefik, M., Three-dimensional computer models of electrospinning systems, Open Physics, 2017, Vol 15, No. 1 pp. 777-789

[7] Mora J., Otín R., Dadvand P., Escolano E., Pasenau M. A., Oñate E., Open tools for electromagnetic simulation programs, The International Journal for Computation and Mathematics in Electrical and Electronic Engineering, 2006, Vol. 25 No. 3, pp. 551-564

[8] Ikhlef N., Mékidèche M.R., Leroy 0 . Kimouche A., 3D electromagnetic simulation of a large diameter cylindrical surface wave excited plasma reactor, The International Journal for Computation and Mathematics in Electrical and Electronic Engineering, 2008, Vol. 27 No. 5, pp. 1069-1080

[9] Yang Z., Becerik-Gerber B., A model calibration framework for simultaneous multi-level building energy simulation, Applied Energy, 2015, Vol. 149, No. 1, pp. 415-431

[10] Solh M., AlRegib G., Characterization of image distortions in multi-camera systems, Proceedings of the 2nd International Conference on Immersive Telecommunications (2009), 2009, Vol. 1, No. 9

[11] Wang Z., Bovik A. C., Sheikh H. R., Simoncelli E. P., Image quality assessment: from error visibility to structural similarity, IEEE Transactions on Image Processing, 2004, Vol. 13 No. 4, pp. 600612

[12] Napieralski P., Kowalczyk M., Detection of vertical disparity in three-dimensional visualizations, Open Physics, 2017, Vol. 15 No. 1, pp. 1028-1033

[13] Wang Z., Shang X., Spatial pooling strategies for perceptual image quality assessment, Proceedings of the International Conference on Image Processing, 2006, Vol. 1, pp. 2945-2948 